\title{
Preoperative serum levels of HE4 and CA125 predict primary optimal cytoreduction in advanced epithelial ovarian cancer: a preliminary model study
}

Li-yuan Feng $\mathbb{D}$, Sheng-bin Liao and Li Li

\begin{abstract}
Objective: The aim of this study is to establish a noninvasive preoperative model for predicting primary optimal cytoreduction in advanced epithelial ovarian cancer by HE4 and CA125 combined with clinicopathological parameters.

Methods: Clinical data including preoperative serum HE4 and CA125 level of 83 patients with advanced epithelial ovarian cancer were collected. The sensitivity, specificity, positive predictive value, negative predictive value and overall accuracy of each clinical parameter were calculated. The Predictive Index score model and the logistic model were constructed to predict the primary optimal cytoreduction.

Results: Optimal surgical cytoreduction was achieved in 62.65\% (52/83) patients. Cutoff values of preoperative serum HE4 and CA125 were $777.10 \mathrm{pmol} / \mathrm{L}$ and $313.60 \mathrm{U} / \mathrm{ml}$. (1) Patients with PIV $\geq 6$ may not be able to achieve optimal surgical cytoreduction. The diagnostic accuracy, NPV, PPV and specificity for diagnosing suboptimal cytoreduction were $71,100,68$, and 100\%, respectively. (2) The logistic model was: logit $p=0.12$ age -2.38 preoperative serum CA125 level -1.86 preoperative serum HE4 level-2.74 histological type-3.37. AUC of the logistic model in the validation group was $0.71(95 \% \mathrm{Cl} 0.54-0.88, P=0.025)$. Sensitivity and specificity were 1.00 and 0.44 , respectively.

Conclusion: Age, preoperative serum CA125 level and preoperative serum HE4 level are important non-invasive predictors of primary optimal surgical cytoreduction in advanced epithelial ovarian cancer. Our PIV and logistic model can be used for assessment before expensive and complex predictive methods including laparoscopy and diagnostic imaging. Further future clinical validation is needed.
\end{abstract}

Keywords: Primary optimal cytoreduction, Noninvasive prediction model, Preoperative HE4 level, Preoperative CA125 level

\section{Introduction}

Ovarian cancer is the most lethal gynecological malignancy. More than $75 \%$ of ovarian cancer patients are in stage III-IV at the time of initial diagnosis. The 5-year survival rate is less than $30 \%$ [1]. Residual lesions after primary surgery remain one of the most important prognostic factors in patients with advanced ovarian cancer [2-5].

\footnotetext{
* Correspondence: lili@gxmu.edu.cn

Department of Gynecologic oncology, Guangxi Medical University Cancer Hospital, 71 Hedi Road, Nanning, Guangxi 530021, People's Republic of China
}

Models predicting the primary optimal surgical debulking are important in guiding the initial treatment of patients with advanced ovarian cancer. Unsatisfactory primary debulking surgery (PDS) does not improve the prognosis, and may increase perioperative morbidity [6, 7]. Advanced ovarian cancer patients who are older, with increased comorbidities, and with a higher disease burden who cannot achieve primary optimal surgical debulking can obtain the similar overall survival rates and lower postoperative adverse events compared to patients with optimal cytoreduction when they choose

(C) The Author(s). 2020 Open Access This article is distributed under the terms of the Creative Commons Attribution 4.0 International License (http://creativecommons.org/licenses/by/4.0/), which permits unrestricted use, distribution, and reproduction in any medium, provided you give appropriate credit to the original author(s) and the source, provide a link to the Creative Commons license, and indicate if changes were made. The Creative Commons Public Domain Dedication waiver (http://creativecommons.org/publicdomain/zero/1.0/) applies to the data made available in this article, unless otherwise stated. 
neoadjuvant chemotherapy [7-10]. Significant variability in the rate of primary optimal surgical cytoreduction for advanced ovarian cancer, ranging from 25 to $90 \%$, exists between institutions [11]. Models predicting the primary optimal surgical debulking are popular, but maddening topic. There are many variables which include tumor volume and stage, patient performance status and ability to tolerate long and complex operations, and surgeons' skills make predictions difficult. Several attempts have been made to predict primary surgical outcome using imaging methods, CA125, and laparoscopic scoring. These studies have been limited by a lack of sensitivity or specificity, complex models, and broad criteria for inclusion.

The ideal preoperative prediction model should accurately identify the optimal and suboptimal surgical outcome, have wide applicability, be accurate and be noninvasive. CA125 and HE4 are considered to be the most promising serum markers of ovarian cancer [12-17]. The purpose of our study was to explore whether the combination of preoperative serum CA125 level and preoperative serum HE4 level could establish a reliable noninvasive preoperative predictive score to assess resectability in initial treatment of advanced ovarian cancer.

\section{Materials and methods}

\section{Study population}

A retrospective analysis of ovarian cancer patients in the Guangxi Medical University Cancer Hospital from January 2012 to December 2018 was performed. The inclusion and exclusion criteria are as follows. Inclusion criteria: 1) patients with complete clinical data (including: age, preoperative serum CA125 level, preoperative serum HE4 level, histological type, FIGO stage, grade, Eastern Cooperative Oncology Group (ECOG) performance status, American Society of Anesthesiologists (ASA) and surgical outcome); 2) patients with ovarian epithelial carcinoma confirmed by pathology; 3) initial treatment of primary surgical debulking; 4) FIGO stage III or IV. Exclusion criteria: 1) patients without complete clinical data; 2) nonovarian epithelial cancer patients confirmed by pathology; 3) patients undergoing preoperative neoadjuvant chemotherapy or a second operation for tumor recurrence; 4) FIGO stage I or II. The patient selection process is shown in Fig. 1. Eighty-three patients met the inclusion criteria. The primary optimal surgical cytoreduction is defined as tumor residue $=0$. All patients provided written informed consent and were approved by the institutional review committee of our hospital.

\section{Statistical analysis}

SPSS17.0 was used for analysis. The comparison between the two groups was performed with a $t$ test (metering data) and a chi square test (classification data). The correlation between surgical outcome and clinicopathological parameters was assessed with a Spearman test. The cut-off value of serum HE4 and CA125 was determined with a ROC curve. Sensitivity was defined as the number of suboptimal surgical cytoreduction patients who were correctly identified divided by the total number of suboptimal surgical cytoreduction patients.

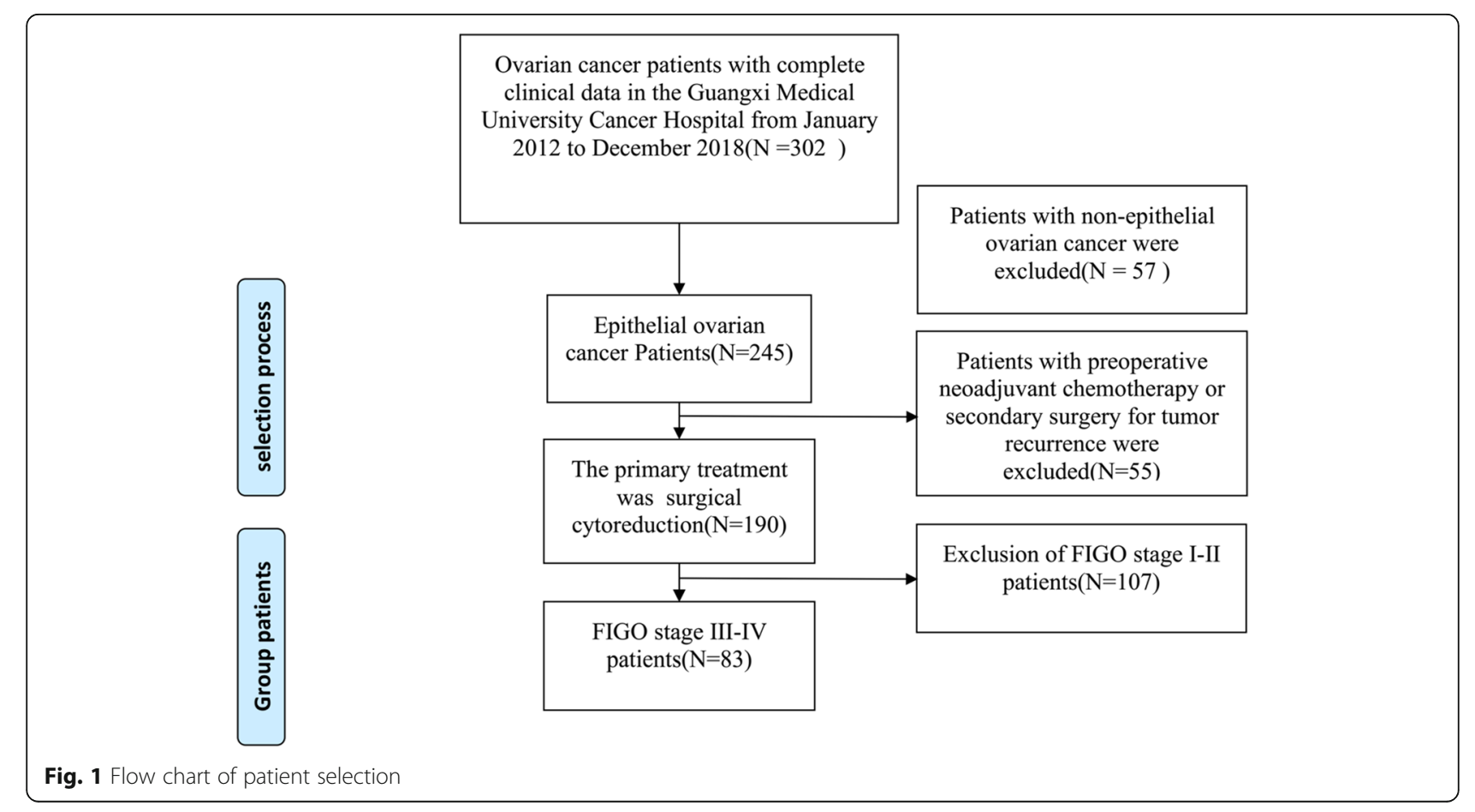


Specificity was defined as the number of optimal surgical cytoreduction patients who were correctly identified divided by the total number of optimal surgical cytoreduction patients. Positive predictive value corresponded to the number of true positives (suboptimal surgical cytoreduction) divided by the total number of patients predicted to have residual disease, and Negative predictive value corresponded to the number of true negatives (optimal surgical cytoreduction) divided by the total number of patients predicted to have no residual disease. Accuracy was calculated as the sum of the true positives and true negatives divided by the total number of patients in the study. Logistic regression analysis was used to find factors associated with optimal debulking and to establish a logistic model. The Hosmer-Leme test was used to assess the goodness of fit. $P$ values were twosided, and $P<0.05$ was considered statistically significant. In order to guarantee an adequate significance of the results, the study power was calculated by PASS11.

\section{Results}

\section{Characteristics of patients}

From January 2012 to December 2018, 83 patients met the inclusion criteria. Optimal surgical cytoreduction was achieved in $62.65 \%(52 / 83)$ of patients, the clinical characteristics of the patients are shown in Table 1 . The mean age of patients was $53.75 \pm 10.51$ years. According to FIGO standard staging, there were 74 cases in stage III and 9 cases in stage IV. 68 (81.93\%) had disease of serous histology; 55(66.27\%)had grade 3. Preoperative serum CA125 level was $1260.84 \pm 1542.01 \mathrm{U} / \mathrm{ml}$, and preoperative serum HE4 level was $635.65 \pm 749.15 \mathrm{pmol} /$ L. $69(83.13 \%)$ of 83 had an ECOG performance status of 0 and ASA class 2; Preoperative serum HE4 level in patients with suboptimal cytoreduction was significantly higher than that in patients with optimal cytoreduction. The differences in age, FIGO stage, grade, histological type, preoperative serum CA125 level, ECOG performance status, and ASA class between optimal cytoreduction and suboptimal cytoreduction groups were not statistically significant.

\section{Diagnostic efficacy of clinicopathological parameters predicting suboptimal cytoreduction}

Spearman correlation analysis showed that surgical outcome was correlated with age, preoperative serum CA125 level and preoperative serum HE4 level (The correlation coefficients were $0.27,0.24$ and 0.41 , respectively). Optimal cytoreduction was more difficult in patients who were older or with higher preoperative serum CA125 and HE4 levels. There was no significant correlation between other clinicopathological parameters and surgical outcome.

Table 1 Clinical characteristics of patients were compared according to surgical outcome

\begin{tabular}{|c|c|c|c|c|}
\hline Clinical characteristics & All (83) & Optimal (52) & Suboptimal (31) & $P$ \\
\hline Age & $53.75 \pm 10.51$ & $52.13 \pm 9.81$ & $56.45 \pm 11.24$ & 0.070 \\
\hline \multicolumn{5}{|l|}{ FIGO stage } \\
\hline III & $74(89.16 \%)$ & $46(88.46 \%)$ & $28(90.32 \%)$ & \multirow[t]{2}{*}{0.791} \\
\hline IV & $9(10.84 \%)$ & $6(11.54 \%)$ & $3(9.68 \%)$ & \\
\hline \multicolumn{5}{|l|}{ Tumor grade } \\
\hline $1-2$ & $28(33.73 \%)$ & $18(34.62 \%)$ & $10(32.26 \%)$ & \multirow[t]{2}{*}{0.826} \\
\hline 3 & $55(66.27 \%)$ & $34(65.38 \%)$ & $21(67.74 \%)$ & \\
\hline \multicolumn{5}{|l|}{ Histology } \\
\hline Serous & $68(81.93 \%)$ & $45(86.54 \%)$ & $23(74.19 \%)$ & \multirow[t]{2}{*}{0.157} \\
\hline Others & $15(18.07 \%)$ & $7(13.46 \%)$ & $8(25.81 \%)$ & \\
\hline Preoperative serum CA125 level(U/ml) & $1260.84 \pm 1542.01$ & $1285.57 \pm 1662.24$ & $1219.36 \pm 1341.36$ & 0.851 \\
\hline Preoperative serum HE4 level (pmol/L) & $635.65 \pm 749.15$ & $419.96 \pm 355.56$ & $997.44 \pm 1050.33$ & $0.006^{*}$ \\
\hline \multicolumn{5}{|l|}{ ECOG performance status } \\
\hline 0 & $69(83.13 \%)$ & $45(86.54 \%)$ & $24(77.42 \%)$ & \multirow[t]{3}{*}{0.131} \\
\hline 1 & 9 (10.84\%) & 6 (11.54\%) & 3 (9.68\%) & \\
\hline 2 & $5(6.03 \%)$ & $1(1.92 \%)$ & 4 (12.90\%) & \\
\hline \multicolumn{5}{|l|}{ ASA } \\
\hline 1 & 9 (10.84\%) & 5 (9.61\%) & 4 (12.90\%) & \multirow[t]{3}{*}{0.886} \\
\hline 2 & $69(83.13 \%)$ & 44 (84.62\%) & 25 (80.65\%) & \\
\hline$\geq 3$ & $5(6.03 \%)$ & $3(5.77 \%)$ & $2(6.45 \%)$ & \\
\hline
\end{tabular}


ROC analysis revealed cut-off values of serum HE4 and CA125 for predicting suboptimal surgical cytoreduction to be $777.10 \mathrm{pmol} / \mathrm{L}$ and $313.60 \mathrm{U} / \mathrm{ml}$. AUC were $0.68(P=0.007)$ and $0.53(P=0.621)$, respectively. We concluded that preoperative serum HE4 was better than CA125 in predicting suboptimal surgical cytoreduction. The sensitivity, specificity, PPV and NPV of each clinical parameter independently predicted suboptimal surgical cytoreduction were shown in Table 2.

PIV model for predicting suboptimal surgical cytoreduction The parameters that meet the accuracy $\geq 75 \%$, PPV $\geq 50 \%$, and $\mathrm{NPV} \geq 50 \%$ are included in the predictive index value (PIV) model, and each parameter is assigned 1 point (Table 2). The PIV of each patient was calculated based on the sum of the parameter weights, with a score ranging from 0 to 8 . The frequency of individual predictive index score was single peak distribution. Two score was peak (Fig. 2a). The frequency distribution of surgical outcome of each score is shown in Fig. 2b. After PIV > 3, the number of suboptimal surgical cytoreduction patients increased, while the number of optimal surgical cytoreduction patients decreased.

The PIV model shows that with the increase of the score, the diagnostic sensitivity becomes lower and the specificity becomes higher (Table 3 ). When patient PIV score $\geq 6$ points, the accuracy, NPV, PPV and specificity for diagnosing suboptimal cytoreduction were $71 \%, 100$, 68 , and $100 \%$. PIV $\geq 6$ (7 cases) predicted that patients who could not achieve optimal cytoreduction did not achieve optimal cytoreduction. PIV $<6$ predicts that 76 patients can achieve optimal cytoreduction, and 52 patients finally achieve optimal cytoreduction.

\section{Logistic model for predicting suboptimal surgical cytoreduction}

Univariate logistic regression results showed that age, preoperative serum HE4 level and preoperative serum CA125 level were the influencing factors for primary optimal surgical cytoreduction. Age $>69$ years $(\mathrm{OR}=$ $0.10,95 \%$ CI $0.01-0.92, P=0.042$ ), preoperative serum
CA125 level $\geq 313.60 \mathrm{U} / \mathrm{ml} \quad(\mathrm{OR}=0.33, \quad 95 \% \quad$ CI $\quad 0.12-$ $0.93, P=0.037$ ), preoperative serum HE4 level $\geq 777.10$ $\mathrm{pmol} / \mathrm{L}(\mathrm{OR}=0.14,95 \%$ CI $0.05-0.42, P=0.000)$ significantly increased the suboptimal cytoreduction. Univariate regression analysis of other clinicopathological factors showed no statistically significant differences.

We constructed a logistic model to predict the optimal surgical cytoreduction. The data was divided into two groups using a random table method (about 1:1): training set $(N=43)$ and verification set $(N=40)$. In the training set, age, FIGO stage, histological type, grade, ECOG performance status, ASA, preoperative serum CA125 level and preoperative serum HE4 level were included to predict the surgical outcome. The results showed that age $>69$ years, preoperative serum CA125 level $\geq 313.60 \mathrm{U} / \mathrm{ml}$, preoperative serum HE4 level $\geq$ $777.10 \mathrm{pmol} / \mathrm{L}$, Serous histological type significantly increased the suboptimal surgical cytoreduction (Age $>69$ years $(\mathrm{OR}=1.13,95 \% \mathrm{CI} 1.02-1.26, P=0.024)$, preoperative serum CA125 level $\geq 313.60 \mathrm{U} / \mathrm{ml}(\mathrm{OR}=0.09,95 \% \mathrm{CI}$ 0.01-0.99, $P=0.049$ ), preoperative serum HE4 level $\geq$ $777.10 \mathrm{pmol} / \mathrm{L}(\mathrm{OR}=0.16,95 \% \mathrm{CI} 0.03-0.98, P=0.047)$, Serous histological type $(\mathrm{OR}=0.07,95 \%$ CI $0.01-0.57$, $P=0.013)$ ). Other clinical parameters were not statistically significant. Logistic regression model was as follows: $\operatorname{logit} p=0.12$ age -2.38 preoperative serum CA125 level-1.86 preoperative serum HE4 level-2.74 histological type-3.37. Hosmer-Leme test showed $P=0.54$, it indicates that the model fits the data better.

In the verification set, the AUC value of logistic model predicting the suboptimal surgical cytoreduction was $0.71(95 \% \mathrm{CI} 0.54-0.88), P=0.025$. The sensitivity and specificity were 1.00 and 0.44 . The ROC curve was shown in Fig. 2c. With a sample size of 83, the study would have $96 \%$ power to meet the AUC $=0.73$ if the optimal surgical cytoreduction rate was $62.65 \%$.

\section{Literature reviews of preoperative serum HE4 predicting the primary optimal surgical cytoreduction}

Almost all studies assessing the predictive value of serum HE4 level in predicting surgical outcome of ovarian cancer

Table 2 Diagnostic efficacy of clinicopathological parameters predicting suboptimal debulking

\begin{tabular}{|c|c|c|c|c|c|c|c|c|c|}
\hline Clinical characteristics & Cutoff & Sensitivity & Specificity & PPV & NPV & Accuracy & AUC & $P$ & Point \\
\hline$\overline{\text { Age }}$ & 68.5 & 0.16 & 0.98 & 0.83 & 0.66 & 0.67 & 0.57 & 0.281 & 2.00 \\
\hline FIGO stage & - & 0.10 & 0.89 & 0.33 & 0.62 & 0.59 & 0.49 & 0.888 & 1.00 \\
\hline Histology & - & 0.26 & 0.87 & 0.53 & 0.66 & 0.64 & 0.56 & 0.349 & 2.00 \\
\hline Tumor grade & - & 0.68 & 0.35 & 0.38 & 0.64 & 0.47 & 0.51 & 0.858 & 1.00 \\
\hline Preoperative serum CA125 level(U/ml) & 313.60 & 0.81 & 0.42 & 0.45 & 0.79 & 0.57 & 0.53 & 0.621 & 1.00 \\
\hline Preoperative serum HE4 level (pmol/L) & 777.10 & 0.48 & 0.89 & 0.71 & 0.74 & 0.73 & 0.68 & $0.007^{*}$ & 2.00 \\
\hline ECOG performance status & 0 & 0.23 & 0.87 & 0.50 & 0.65 & 0.63 & 0.55 & 0.489 & 2 \\
\hline ASA & 3 & 0.07 & 0.94 & 0.40 & 0.63 & 0.61 & 0.50 & 0.959 & 1 \\
\hline
\end{tabular}

* means $P<0.05$ 

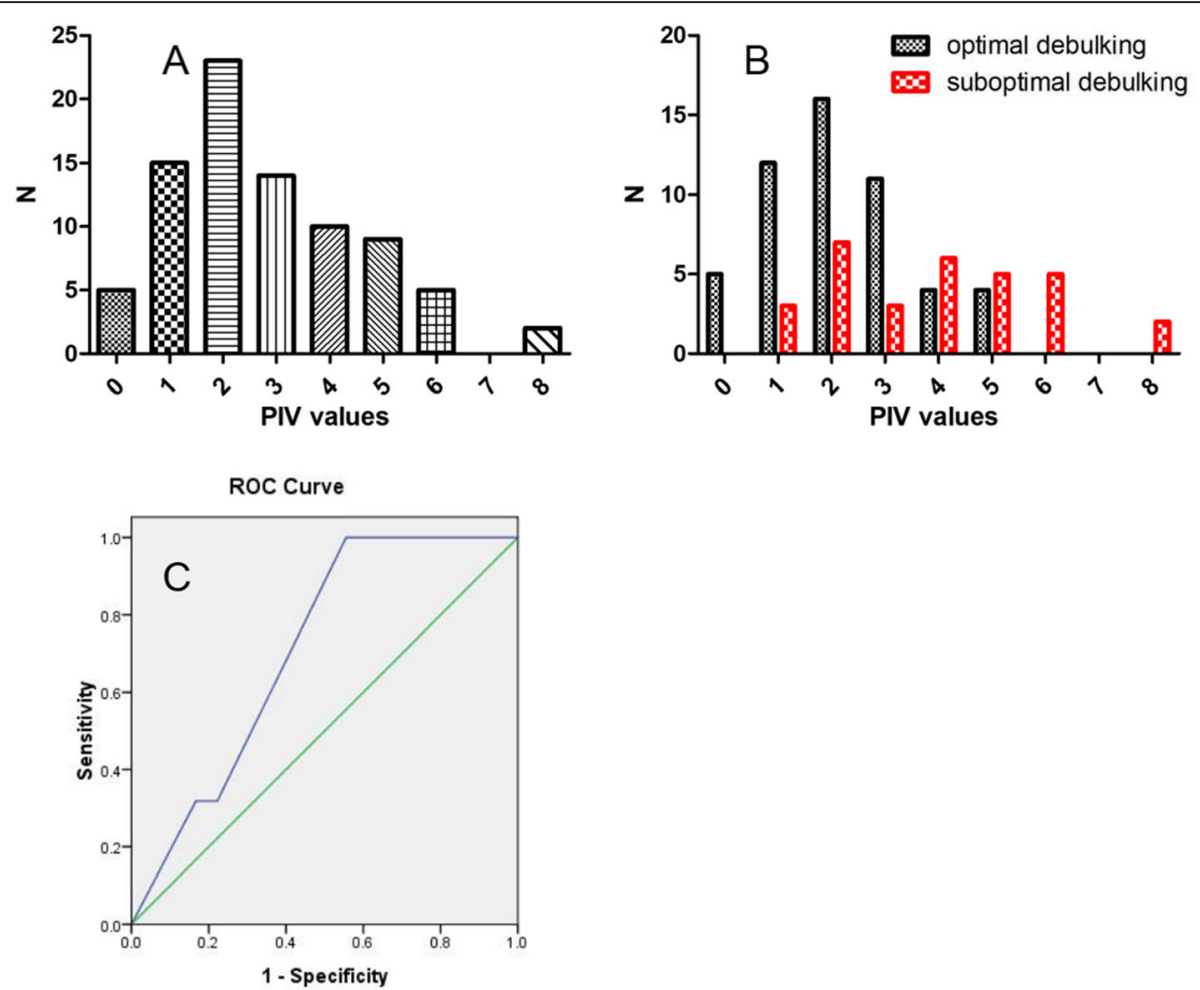

Fig. 2 a: Number frequency of PIV model score. b: Relationship between PIV model score and optimal debulking. c: The ROC curve of logistic model in verification set

have confirmed the effectiveness of HE4. We reviewed the study of HE4 in predicting the primary optimal surgical cytoreduction, and compared the diagnostic efficacy of each study. Results are shown in Table 4.

\section{Discussion}

Recurrence of ovarian cancer after surgery and first line chemotherapy remains a problem. Recurrant oviarian cancer typically poorly responds to first, and sometimes even second line chemotherapy. In this scenario, it possible that the ovarian cancer inherent resistance may be due to reduced immunosurveillance and drugresistant cells $[18,19]$. Surgical removal of drug-resistant tumors can obtain the maximum clinical benefit. Recent

Table 3 Diagnostic efficacy of each value of PIV model

\begin{tabular}{llllll}
\hline PIV & Sensitivity(\%) & Specificity(\%) & NPV(\%) & PPV(\%) & Accuracy(\%) \\
\hline$\geq 1$ & 100 & 10 & 40 & 100 & 43 \\
$\geq 2$ & 90 & 33 & 47 & 100 & 54 \\
$\geq 3$ & 68 & 63 & 53 & 77 & 65 \\
$\geq 4$ & 58 & 85 & 69 & 77 & 75 \\
$\geq 5$ & 39 & 92 & 75 & 72 & 72 \\
$\geq 6$ & 23 & 100 & 100 & 68 & 71 \\
$\geq 8$ & 6.5 & 100 & 100 & 64 & 65 \\
\hline
\end{tabular}

advanced robotic approaches increase efficacy of safe surgical debulking of early-stage ovarian cancer. Currently the majority of patients with FIGO III-IV are staged by laparotomy [20, 21]. Non-invasive biomarkers and imaging indicators are the first choice for the prediction model of advanced ovarian cancer patients. Imaging combined with physical examination and health assessment is a common indicator for clinicians to judge whether advanced ovarian cancer patients can perform ideal tumor cytoreductive surgery. However, the systematic review shows that the CT-based predictive model does not have good sensitivity and specificity on surgical residual lesions and the CT model is complex and difficult to apply to clinical practice [22].

It has been thought in the past that higher preoperative serum CA125 levels are directly related to a larger tumor burden, and accurate CA125 cut-off values can help distinguish whether ovarian tumor patients can achieve optimal surgical cytoreduction. But based on studies over the past 10 years, there is no uniform conclusion as to whether preoperative CA125 level can predict primary optimal surgical cytoreduction [23-25]. Most studies have shown that 500 $\mathrm{U} / \mathrm{ml}$ is the appropriate cut off value for CA125, but not practical in clinical applications. The study reported that the AUC of preoperative CA125 predictive optimal surgical cytoreduction was $0.57-0.83$ [26-30], prediction efficiency 
Table 4 Literature reviews of HE4 predictive diagnostic efficacy for primary optimal surgical debulking

\begin{tabular}{llllllllllllll}
\hline Year & Author & $N$ & Country & $\begin{array}{l}\text { Optimal surgical } \\
\text { debulking rate }\end{array}$ & $\begin{array}{l}\text { HE4 cutoff } \\
\text { value (pmol/L) }\end{array}$ & Sensitivity & Specificity & PPV & NPV & AUC & statistical method References \\
\hline 2016 & Karlsen & 150 & Danish & $27.00 \%$ & 262.00 & - & - & - & - & 0.79 & logistic & \\
2012 & Angioli & 57 & Italy & $66.70 \%$ & 262.00 & $86.10 \%$ & $89.50 \%$ & $93.90 \%$ & $77.00 \%$ & 0.86 & ROC & $1[18]$ \\
2012 & Braicu & 275 & Germany & $68.40 \%$ & 235.00 & $76.60 \%$ & $47.30 \%$ & - & - & 0.63 & ROC & $1[19]$ \\
2013 & Braicu & 275 & Germany & $68.50 \%$ & 500.00 & $51.90 \%$ & $70.40 \%$ & - & - & 0.63 & ROC & $1[20]$ \\
2016 & Shen & 39 & China & - & 353.22 & $77.40 \%$ & $75.00 \%$ & $92.30 \%$ & $46.20 \%$ & 0.76 & ROC & $1[21]$ \\
2015 & Tang & 90 & China & $47.70 \%$ & 473.00 & $81.00 \%$ & $56.00 \%$ & $67.00 \%$ & $73.00 \%$ & 0.72 & ROC & $1[22]$ \\
2017 & Paunovic & 50 & Serbia & $44.00 \%$ & 413.00 & - & - & - & - & - & logistic & $1[23]$ \\
2014 & Glaz & 56 & Poland & $45.00 \%$ & 218.43 & $86.60 \% ;$ & $91.30 \%$ & $92.90 \%$ & $84.00 \%$ & - & ROC & $1[24]$ \\
\hline
\end{tabular}

is either weak or high $[25,31,32]$. There are few studies on HE4 in predicting primary optimal surgical cytoreduction of advanced ovarian cancer, but almost all studies assessing the predictive value of serum HE4 level in predicting surgical outcome for ovarian cancer have confirmed the effectiveness of HE4.

Our study shows that preoperative serum CA125 combined with preoperative serum HE4 can predict whether advanced ovarian cancer patients can achieve optimal surgical cytoreduction. The advantage of this study is that the latest standard $\mathrm{RD}=0$ is used as the definition of primary optimal surgical cytoreduction, only patients with advanced ovarian cancer are included, and predictive models have been developed from two different methods (PIV and logistic regression) for easy understanding and management. Important clinical variables were also considered: combined with preoperative serum CA125 and HE4. In fact, predictive models including age, FIGO stage, histological type, grade, preoperative serum CA125 level, and preoperative serum HE4 level were more accurate than the CA125 model alone.

Accumulating evidence suggests that the management of ovarian cancer should be personalized taking into account the performance status of the patient, in particular in case of elderly women [33, 34]. We included several important preoperative non-invasive parameters (age, FIGO stage, histological type, grade, preoperative serum CA125 level, preoperative serum HE4 level, ECOG performance status, and ASA). The accuracy of inclusion parameters in predicting whether advanced ovarian cancer patients can achieve optimal surgical cytoreduction is $0.49-0.68$. The diagnostic accuracy, NPV, PPV, specificity of PIV model was $71,100,68,100 \%$. Negative predictive value represent a very important clinical parameter and are the proportion of patients who are considered to have unresectable disease and do not actually achieve optimal surgical cytoreduction. Negative predictive value of $100 \%$ can avoid unnecessary laparotomy exploration.

In 2006, Fagotti et al. [35] quantified the lesion information obtained under laparoscopic exploration, and predicted the statistical probability of the surgical outcome according to various factors, seven laparoscopic features were selected for inclusion in the PIV model. When PIV $\geq 8$ points, the specificity, PPV, and NPV of the model was 100,100 , and $70 \%$. This model has been validated later. However, laparoscopy also has certain limitations. In fact, as the disease advances, technical limits due to the absence of a direct tactile evaluation by palpation and the presence of fixed masses and carcinomatous adhesions hindering the visualization of certain anatomical spaces are intuitive. For patients who can not achieve optimal surgical cytoreduction, laparoscopic exploration exposes them to surgical complications, delays the onset of chemotherapy, and does not yield prognostic benefits.

Since Fagotti's laparoscopic PIV model has a 100\% positive predictive value, the negative predictive value of our study is $100 \%$. The evaluation model of this study suggests that patients who can achieve optimal surgical cytoreduction may benefit from the second laparoscopic evaluation. Those patients who are judged to be resectable by the inexpensive, simple and non-invasive preoperative prediction model in our study can be re-evaluated by a more expensive and complex laparoscopic model. This secondary evaluation method can improve the expected surgical outcome and reduce the rate of inappropriate exploration. Similarly, the logistic model for predicting surgical outcome with age, preoperative serum CA125 level and preoperative serum HE4 level also has certain predictive efficacy. Like the PIV model, it confirms that the combination of preoperative serum CA125 level and preoperative serum HE4 level can establish a reliable preoperative non-invasive predictive model. This reliable non-invasive preoperative predictive model can be used before expensive and complex predictive methods such as laparoscopy or imaging.

There are several shortcomings in this study. First, like other models for predicting whether ovarian cancer patients can achieve optimal surgical cytoreduction, this study is a retrospective analysis. Second, the number of 
samples is small, and the model therefore needs further external validation. Third, resectability is strictly correlated to surgeons' skills. This variable is difficult to ascertain and was not included in our model.

In conclusion, our study demonstrates in the initial treatment plan for advanced ovarian cancer patients, the diagnostic accuracy of age, preoperative serum CA125 level and preoperative serum HE4 level in assessing optimal surgical cytoreduction should be considered. They can be used as a pre-evaluation before laparoscopy or diagnostic imaging prediction models.

\section{Acknowledgements}

Not applicable.

\section{Authors' contributions}

First author: $\mathrm{L}-\mathrm{y} F$ responsible for statistical analysis of data and writing articles. Second author: S-bL responsible for statistical analysis of data. Corresponding author: professor LL responsible for the revision. All authors read and approved the final manuscript.

\section{Funding}

This work was supported by grants from the National Natural Science Foundation of China (Grant No. 81572579, No. 81660430). The funder had no role in study design, data collection and analysis, decision to publish, or preparation of the manuscript.

\section{Availability of data and materials}

Not applicable.

\section{Ethics approval and consent to participate}

The research on human genetic resources materials in the "Preoperative scrum levels of HE4 and CA125 predict primary optimal debulking in advanced epithelial ovarian cancer: a preliminary model study" by Feng Liyuan was reviewed by the Ethics Committee of the affiliated tumor hospital of Guangxi Medical University, and it was considered that the study met the requirements of medical ethics (Number: LW2019029).

\section{Consent for publication}

Not applicable.

\section{Competing interests}

The authors declare that they have no competing interests.

Received: 10 October 2019 Accepted: 22 January 2020

Published online: 12 February 2020

\section{References}

1. Siegel RL, Miller KD, Jemal A. Cancer statistics, 2017. CA Cancer J Clin. 2017; 67(1):7-30.

2. Melamed A, Manning-Geist B, Bregar AJ, et al. Associations between residual disease and survival in epithelial ovarian cancer by histologic type. Gynecol Oncol. 2017;147(2):250-6.

3. Sehouli J, Grabowski JP. Surgery for recurrent ovarian cancer: options and limits. Best Pract Res Clin Obstet Gynaecol. 2017:41:88-95.

4. Luo Y, Lee M, Kim HS, et al. Effect of neoadjuvant chemotherapy on platinum resistance in stage IIIC and IV epithelial ovarian cancer. Medicine. 2016;95(36): 4797

5. Fagotti A, Vizzielli G, Fanfani F, et al. Introduction of staging laparoscopy in the management of advanced epithelial ovarian, tubal and peritoneal cancer: impact on prognosis in a single institution experience. Gynecol Oncol. 2013;131(2):341-6.

6. Pepin $\mathrm{K}$, Bregar $\mathrm{A}$, Davis $\mathrm{M}$, et al. Intensive care admissions among ovarian cancer patients treated with primary debulking surgery and neoadjuvant chemotherapy-interval debulking surgery. Gynecol Oncol. 2017;147(3):612-6.

7. Gill SE, McGree ME, Weaver AL, et al. Optimizing the treatment of ovarian cancer: Neoadjuvant chemotherapy and interval debulking versus primary debulking surgery for epithelial ovarian cancers likely to have suboptimal resection. Gynecol Oncol. 2017;144(2):266-73.

8. Makar AP, Tropé CG, Tummers P, et al. Advanced ovarian cancer: primary or interval Debulking? Five categories of patients in view of the results of randomized trials and tumor biology: primary Debulking surgery and interval Debulking surgery for advanced ovarian cancer. Oncologist. 2016; 21(6):745-54.

9. Cho JH, Kim S, Song YS. Neoadjuvant chemotherapy in advanced ovarian cancer: optimal patient selection and response evaluation. Chin Clin Oncol. 2018;7(6):58.

10. Baek M-H, Lee S-W, Park J-Y, et al. Preoperative predictive factors for complete Cytoreduction and survival outcome in epithelial ovarian, tubal, and peritoneal cancer after Neoadjuvant chemotherapy. Int J Gynecol Cancer. 2017;27(3):420-9

11. Bristow RE, Tomacruz RS, Armstrong DK, et al. Survival effect of maximal cytoreductive surgery for advanced ovarian carcinoma during the platinum era: a meta-analysis. J Clin Oncol. 2002;20(5):1248-59.

12. Lycke M, Kristjansdottir B, Sundfeldt K. A multicenter clinical trial validating the performance of $\mathrm{HE4}, \mathrm{CA} 125$, risk of ovarian malignancy algorithm and risk of malignancy index. Gynecol Oncol. 2018;151(1):159-65.

13. Moore RG, Blackman A, Miller MC, et al. Multiple biomarker algorithms to predict epithelial ovarian cancer in women with a pelvic mass: can additional makers improve performance? Gynecol Oncol. 2019;154:150-5.

14. Dewan $R$, Dewan $A$, Jindal $M$, et al. Diagnostic performance of serum human epididymis protein 4 (HE4) for prediction of malignancy in ovarian masses. Asian Pac J Cancer Prev. 2019;20(4):1 103-8.

15. Ahmed AA, Abdou AM. Diagnostic accuracy of CA125 and HE4 in ovarian carcinoma patients and the effect of confounders on their serum levels. Curr Probl Cancer. 2019;43:450-60.

16. Plotti F, Guzzo F, Schiro T, et al. Role of human epididymis protein 4 (HE4) in detecting recurrence in CA125 negative ovarian cancer patients. Int J Gynecol Cancer. 2019. https://doi.org/10.1136/ijgc-2019-000211.

17. Wang $Q$, Wu Y, Zhang H, et al. Clinical Value of Serum HE4, CA125, CA72-4, and ROMA Index for Diagnosis of Ovarian Cancer and Prediction of Postoperative Recurrence. Clin Lab. 2019;65(4). https://doi.org/10.7754/Clin. Lab.2018.181030.

18. Lagana AS, Sofo V, Vitale SG, et al. Epithelial ovarian cancer inherent resistance: may the pleiotropic interaction between reduced immunosurveillance and drug-resistant cells play a key role? Gynecol Oncol Rep. 2016;18:57-8.

19. Giannakeas V, Sopik V, Narod SA. A model for ovarian cancer progression based on inherent resistance. Gynecol Oncol. 2016;142(3):484-9.

20. Bellia A, Vitale SG, Lagana AS, et al. Feasibility and surgical outcomes of conventional and robot-assisted laparoscopy for early-stage ovarian cancer: a retrospective, multicenter analysis. Arch Gynecol Obstet. 2016;294(3):615-22.

21. Minig L, Padilla Iserte $P$, Zorrero $C$, et al. Robotic surgery in women with ovarian cancer: surgical technique and evidence of clinical outcomes. J Minim Invasive Gynecol. 2016;23(3):309-16.

22. Rutten MJ, van de Vrie $R$, Bruining $A$, et al. Predicting surgical outcome in patients with International Federation of Gynecology and Obstetrics Stage III or IV ovarian cancer using computed tomography. Int J Gynecol Cancer. 2015;25(3):407-15.

23. Rutten MJ, van de Vrie R, Bruining $A$, et al. Predicting surgical outcome in patients with International Federation of Gynecology and Obstetrics stage III or IV ovarian cancer using computed tomography: a systematic review of prediction models. Int J Gynecol Cancer. 2015;25(3):407-15.

24. Vorgias G, lavazzo C, Savvopoulos P, et al. Can the preoperative Ca-125 level predict optimal cytoreduction in patients with advanced ovarian carcinoma? A single institution cohort study. Gynecol Oncol. 2009;112(1):11-5.

25. Kang S, Kim TJ, Nam BH, et al. Preoperative serum CA-125 levels and risk of suboptimal cytoreduction in ovarian cancer: a meta-analysis. J Surg Oncol. 2010;101(1):13-7.

26. Modares Gilani M, Karimi Zarchi M, Ghaemmaghami F, et al. RETRACTED: a study to evaluate the utility of presurgical CA125 to predict optimal tumor cytoreduction of epithelial ovarian cancer. Gynecol Oncol. 2007;105(3):780-3.

27. Gemer O, Lurian M, Gdalevich M, et al. A multicenter study of CA 125 level as a predictor of non-optimal primary cytoreduction of advanced epithelial ovarian cancer. Eur J Surg Oncol. 2005;31(9):1006-10.

28. Arits AH, Stoot JE, Botterweck AA, et al. Preoperative serum CA125 levels do not predict suboptimal cytoreductive surgery in epithelial ovarian cancer. Int J Gynecol Cancer. 2008;18(4):621-8. 
29. Chudecka-Glaz AM, Cymbaluk-Ploska AA, Menkiszak JL, et al. Serum HE4,

CA125, YKL-40, bcl-2, cathepsin-L and prediction optimal debulking surgery, response to chemotherapy in ovarian cancer. J Ovarian Res. 2014;7:62.

30. Barlow TS, Przybylski M, Schilder JM, et al. The utility of presurgical CA125 to predict optimal tumor cytoreduction of epithelial ovarian cancer. Int J Gynecol Cancer. 2006;16(2):496-500.

31. Cooper BC, Sood AK, Davis CS, et al. Preoperative CA 125 levels: an independent prognostic factor for epithelial ovarian cancer. Obstet Gynecol. 2002;100(1):59-64

32. Memarzadeh S, Lee SB, Berek JS, et al. CA125 levels are a weak predictor of optimal cytoreductive surgery in patients with advanced epithelial ovarian cancer. Int J Gynecol Cancer. 2003;13(2):120-4.

33. Vitale SG, Capriglione S, Zito G, et al. Management of endometrial, ovarian and cervical cancer in the elderly: current approach to a challenging condition. Arch Gynecol Obstet. 2019:299(2):299-315.

34. Schuurman MS, Kruitwagen R, Portielje JEA, et al. Treatment and outcome of elderly patients with advanced stage ovarian cancer: a nationwide analysis. Gynecol Oncol. 2018;149(2):270-4.

35. Fagotti A, Ferrandina G, Fanfani F, et al. A laparoscopy-based score to predict surgical outcome in patients with advanced ovarian carcinoma: a pilot study. Ann Surg Oncol. 2006;13(8):1156-61

\section{Publisher's Note}

Springer Nature remains neutral with regard to jurisdictional claims in published maps and institutional affiliations.

Ready to submit your research? Choose BMC and benefit from:

- fast, convenient online submission

- thorough peer review by experienced researchers in your field

- rapid publication on acceptance

- support for research data, including large and complex data types

- gold Open Access which fosters wider collaboration and increased citations

- maximum visibility for your research: over $100 \mathrm{M}$ website views per year

At BMC, research is always in progress.

Learn more biomedcentral.com/submissions 